\title{
Survival Benefit for Patients With Metastatic Urothelial Carcinoma Receiving Continuous Maintenance Chemotherapy
}

\author{
CHUANG-CHI LIAW $^{1}$, TZU-YAO LIAO ${ }^{1}$, KE-HUNG TSUI $^{2}$ and YU-HSIANG JUAN ${ }^{3}$ \\ ${ }^{1}$ Division of Hemato-Oncology, Department of Internal Medicine Chang-Gung Memorial Hospital \\ and Chang-Gung University College of Medicine, Taoyuan, Taiwan, R.O.C.; \\ ${ }^{2}$ Department of Urology, Chang-Gung Memorial Hospital and Chang-Gung \\ University College of Medicine, Taoyuan, Taiwan, R.O.C.; \\ ${ }^{3}$ Department of Medical Imaging and Intervention, Chang-Gung Memorial Hospital \\ and Chang-Gung University College of Medicine, Taoyuan, Taiwan, R.O.C.
}

\begin{abstract}
Background/Aim: Urothelial carcinoma is a chemo-sensitive cancer. We investigated the contributory factors to survival benefit of metastatic urothelial carcinoma (MUC) patients receiving continuous maintenance chemotherapy. Patients and Methods: Inclusion criteria were: i) pathology-confirmed urothelial carcinoma, ii) metastatic lesions identified mainly on pre-therapy computed tomography (CT) scans, and iii) inpatient-administered chemotherapy of at least three cycles. Chemotherapy regimens included 5-fluorouracil, leucovorin, cisplatin, and gemcitabine. Results: A total of 139 cases were enrolled in this study. The overall objective response rate was $60 \%$ and the median survival time was 17 months. Eight-two (59\%) patients had inflammation-related symptoms following the course of chemotherapy. Fifty-five (41\%) patients survived more than two years. All patients exhibited various fibrosis formations. No patient experienced unfavorable metastatic conditions. Inflammation-related symptoms remained in 28 (51\%) patients. We found that surgery, invasive procedures, and infection likely led to a rapid tumor progression. Conclusion: Continuous maintenance chemotherapy targeting chemo-sensitive tumors, administered at metronomic intervals and focus on tumor microenvironment, can increase MUC survival benefits.
\end{abstract}

This article is freely accessible online.

Correspondence to: Chuang-Chi Liaw, Division of HematoOncology, Department of Internal Medicine, Chang-Gung Memorial Hospital, 5 Fusing St., Gueishan Township, Taoyuan City, 333, Taiwan, R.O.C. Tel: +886 33281200 ext 8825, Fax: +8863278211 , e-mail: e102309@adm.cgmh.org.tw ORCID: 0000-0001-6833-9121

Key Words: Urothelial carcinoma, metastases, chemotherapy, maintenance chemotherapy inflammation, computed tomography.
Urothelial carcinoma is a chemo-sensitive cancer. The median overall survival (OS) time of metastatic urothelial carcinoma (MUC) patients is 17 months following systemic chemotherapy according to a Japanese study (1). In our previous report of bladder urothelial carcinoma, with peritoneal involvement receiving continuous maintenance chemotherapy, the median OS time was 28 months (2).

Inflammatory cytokines are important mediators of MUC $(3,4)$. Transforming growth factor-beta (TGF- $\beta$ ) signaling activity promotes cancer progression at multiple stages of the metastatic process, including the epithelial-to-mesenchymal transition (EMT) (5). Production of inflammatory cytokines can be due to cancer itself and/or from host reactions, such as invasive procedures, therapy, or infection. Increased production of inflammatory cytokines can result in thromboembolic complications, cancer cachexia, and tumor progression, which can carry poor prognosis $(3,4,6)$. The main thromboembolic complications are cerebral thrombosis and pulmonary vein obstructive syndrome (PVOS) $(3,4)$.

In this study, we retrospectively investigated patients with MUC receiving continuous maintenance chemotherapy for survival benefit. We also analyzed the contributory factors to their survival time over two years and assessed their potential for long-term survival.

\section{Patients and Methods}

This study was approved by the Institutional Review Board (the "IRB") of Chang Gung Medical Foundation on 2018/08/27. The IRB is organized and operates according to the guidelines of good clinical practice and the applicable laws and regulations (IRB No.: 2018013198B0).

Study population. A retrospective study was conducted using data collected from MUC patients who were admitted to the oncology wards of the Chang-Gung Memorial Hospital in Taoyuan, Taiwan, between January 2008 and December 2017. All patients were 
hospitalized due to complications following chemotherapy treatment and received palliative care. Computed tomography (CT) scans were performed in all cases to evaluate the extent of the tumor. A medical oncologist specializing in urological cancer provided most of the data. The criteria for inclusion were: i) pathology-confirmed urothelial carcinoma, ii) metastatic lesions, including peritoneum, lymph node (LN), lung, bone, and liver metastases as identified mainly by pre-therapy CT scans, and iii) needing at least three cycles of inpatient-administered chemotherapy. Regional, iliac, paraaortic, supraclavicular, and inguinal $\mathrm{LN}$ involvement were all defined as LN metastases.

Chemotherapy regimens included: i) 5-fluorouracil, ii) leucovorin, iii) cisplatin, and iv) gemcitabine (Gemmis; TTY, Taipei, Taiwan, ROC). The original dosage was $50 \mathrm{mg} / \mathrm{m}^{2}$ cisplatin by intravenous infusion for 3 hours on day 1 , plus $1000 \mathrm{mg} / \mathrm{m}^{2}$ gemcitabine by intravenous infusion for 30 minutes on days 1,15 , and 22 , in combination with a continuous intravenous infusion of 5 -fluorouracil and leucovorin at $1000 \mathrm{mg} / \mathrm{m}^{2}$ and $100 \mathrm{mg}$, respectively, for 46 hours, every 28 days. If renal function impairment was observed (serum creatinine value $>2 \mathrm{mg} / \mathrm{dl}$ ), cisplatin was changed to carboplatin $\left(150 \mathrm{mg} / \mathrm{m}^{2}\right)$ by intravenous infusion for 2 hours. The schedule could be modified during the course of chemotherapy. In general, we administered an inpatient chemotherapy regimen of 5-fluorouracil and leucovorin plus cisplatin with or without the gemcitabine every four weeks, and gemcitabine alone was usually administered in the outpatient department every two weeks. Treatment strategies were initially aggressive, but were slowed down based on stable or improving conditions and depending on the results of image studies. We extended the chemotherapy interval or administered gemcitabine only if the patient was too old, had a severe comorbidity, or a poor performance status. Continuous maintenance chemotherapy was administered until tumor progression was no longer detected or the patient or doctor decided to discontinue therapy. Only a few cases received 2nd line chemotherapy. The regimen was 5-fluorouracil, leucovorin, and mitomycin. Follow-up CT scans were performed every three months to assess tumor response in most patients. We also recorded the number of inpatient and outpatient chemotherapy sessions separately. Objective tumor response to chemotherapy was defined as tumor response based on RECIST guidelines (7), and/or obvious decrease in peritoneal thickness, suspicion of 'dirty' peritoneal spread necrosis, and/or osteoblastic change or regression of osteolytic lesions.

Identification of inflammation and inflammation-related symptoms in MUC patients during the clinical treatment was necessary. Inflammation-related symptoms were defined as symptoms related to inflammation caused by the tumor and/or host reaction. These included thromboembolism complications such as PVOS, cerebral thrombosis and cachexia, and neoplastic fever. Enoxaparin (Sanofi-Aventis) and dexamethasone were administered to treat thromboembolism complications, while progesterone agents and naproxen were administered for cancer cachexia and neoplastic fever, respectively $(3,4,8,9)$.

Analysis of survival time for more than two years. For the analysis of the contributory factors to patients' survival time over two years and their potential for long-term survival, we report interesting cases and their associated images. We analyzed: i) metastatic conditions, ii) objective tumor response to chemotherapy, iii) formation of fibrosis, and iv) occurrence of symptoms related to inflammation.
These findings were all based on CT scans and clinical manifestations during the clinical course of therapy. We defined multiple ( $>4)$ bone, lung, and liver metastases as unfavorable metastatic conditions $(4,10)$. Oligo-metastases $(\leq 4)$ were defined as limited and favorable metastatic lesions (11).

Tumor response following chemotherapy led to a wound-like healing process and fibrosis formation within the tumor (12). Fibrosis and healing were defined as the detection of fibrosis formation, an obvious decrease of peritoneal lesion with peritoneal thickness, and 'dirty' or regressing osteolytic lesions with osteoblastic change following chemotherapy from the CT scan diagnosis. Patients who were potentially cured were defined as having tumor regression following chemotherapy in addition to withdrawal from chemotherapy for more than one year.

Statistical methods. Continuous data (presented as mean \pm standard deviation) were used to determine the number of chemotherapy cycles delivered by inpatient and outpatient services. The survival time was calculated from the start of chemotherapy to death and survival curves were determined using the Kaplan-Meier method. Significant differences between survival curves were measured using the log-rank test.

\section{Results}

Metastatic urothelial carcinoma, treatment and patient survival. From the reviewed data, 139 consecutive MUC patients met the inclusion criteria, involving 90 men and 49 women (median age 66 years, range: 40-86 years). The patients' clinical characteristics are shown in Table I. The cancer types were bladder $(n=88)$ and upper urinary tract $(n=51)$, which included renal pelvis $(n=34)$ and ureter $(n=17)$. Before chemotherapy, 130 patients had peritoneal involvement as the most common metastatic site. Other metastatic sites were: i) lung $(n=39)$, ii) LNs $(n=30)$, iii) bone $(n=8)$, and iv) liver $(n=5)$. Multiple $(>4)$ cases of $i)$ lung $(n=21)$, ii) bone $(n=3)$, and iii) liver $(n=4)$ metastases were noted. Performance status of patients was 0-1 for all. Multiple primary sites of urothelial carcinoma included: i) bladder $(n=3)$ and ii) upper urinary tract cancer $(n=10)$, including renal pelvis $(n=8)$ and ureter $(n=2)$. All other primary sites were localized tumors. Before chemotherapy, 26 upper urinary tract cancer patients received nephrectomy and/or ureterectomy, 23 bladder cancer patients received radical or partial cystectomy, and 10 bladder cancer patients received concomitant chemoradiation therapy (CCRT), including 6 during initial therapy and 4 following cystectomy. Three cases (two bladder and one ureter cancer) had previous renal transplantation.

The mean number of inpatient chemotherapy cycles was $10 \pm 15$ (range: $3-34$ cycles) and the mean number of

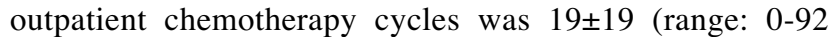
cycles) for all patients. The overall objective response rate in all patients was $60 \%$, with $67 \%$ in the bladder and $49 \%$ in the upper urinary tract. The side effects of chemotherapy were mild and tolerated. Symptoms related to inflammation 
Table I. Clinical characteristics, objective tumor response, and survival time of 139 patients with metastatic urothelial carcinoma receiving chemotherapy.

\begin{tabular}{|c|c|c|c|}
\hline Characteristics & $\begin{array}{l}\text { Total patients } \\
\quad(\mathrm{n}=139)\end{array}$ & $\begin{array}{c}\text { Bladder } \\
(\mathrm{n}=88)\end{array}$ & $\begin{array}{l}\text { Upper urinary tract }(n=51 \\
\text { renal pelvis } 34 \text {, ureter } 17)\end{array}$ \\
\hline Age (years), median (range) & $66(40-86)$ & $66(40-86)$ & $66(42-81)$ \\
\hline Gender (Male/Female) & $90 / 49$ & $63 / 25$ & $27 / 24$ \\
\hline Metastatic site: Peritoneum/Lung/LN/Bone/Liver & $130 / 39 / 30 / 8 / 5$ & $86 / 25 / 12 / 2 / 4$ & $44 / 14 / 18 / 6 / 1$ \\
\hline No. of inpatient chemotherapy, mean \pm SD (range) & $10 \pm 15(3-34)$ & $10 \pm 6(3-34)$ & $11 \pm 6(3-27)$ \\
\hline No. of outpatient chemotherapy, mean \pm SD (range) & $19 \pm 19(0-92)$ & $18 \pm 19(0-92)$ & $22 \pm 18(0-85)$ \\
\hline Objective tumor response rate & $60 \%$ & $67 \%$ & $49 \%$ \\
\hline $\begin{array}{l}\text { Inflammation-related symptoms, } \\
\text { Yes/No No. of patients }(\%)\end{array}$ & $82(59 \%) / 57(41 \%)$ & $43(49 \%) / 45(51 \%)$ & $39(76 \%) / 12(24 \%)$ \\
\hline OS median, months (range) & $17(2-126)$ & $18(3->125)$ & $17(2-126)$ \\
\hline 2-yr/3-yr/5-yr survival, No. of patients (\%) & $55(41 \%) / 31(26 \%) / 12(18 \%)$ & $31(39 \%) / 17(24 \%) / 8(20 \%)$ & $24(44 \%) / 14(29 \%) / 4(16 \%)$ \\
\hline Death/Alive/Lost, No. of patients (\%) & $107(77 \%) / 24(17 \%) / 8(6 \%)$ & $65(74 \%) / 17(19 \%) / 6(7 \%)$ & $42(82 \%) / 7(14 \%) / 2(4 \%)$ \\
\hline $\begin{array}{l}\text { Cause of death: cons. distur./resp. } \\
\text { fail./tumor bleeding, No. of patients (\%) }\end{array}$ & $82(77 \%) / 24(22 \%) / 1(1 \%)$ & $53(82 \%) / 12(18 \%) / 0(0 \%)$ & $29(69 \%) / 12(29 \%) / 1(2 \%)$ \\
\hline
\end{tabular}

LN: Lymph node; SD: standard deviation; OS: overall survival; yr: year; cons. distur.: consciousness disturbance; resp. fail.: respiratory failure.

Table II. Clinical characteristics of 55 patients with metastatic urothelial carcinoma receiving chemotherapy with a survival time of more than two years.

\begin{tabular}{|c|c|c|c|}
\hline Characteristics & $\begin{array}{l}\text { Total patients } \\
\quad(\mathrm{n}=55)\end{array}$ & $\begin{array}{c}\text { Bladder } \\
(\mathrm{n}=31)\end{array}$ & $\begin{array}{l}\text { Upper urinary tract } \\
\qquad(\mathrm{n}=24)\end{array}$ \\
\hline Gender (years), median (range) & $66(40-85)$ & $67(40-85)$ & $66(42-81)$ \\
\hline Sex (Male/Female) & $38 / 17$ & $26 / 5$ & $12 / 12$ \\
\hline $\begin{array}{l}\text { Metastatic site: Peritoneum only/Peritoneum+Lung/ } \\
\text { Peritoneum+ LN /Peritoneum + bone/Bone only }\end{array}$ & $35 / 7 / 9 / 3 / 1$ & $25 / 4 / 2 / 0 / 0$ & $10 / 3 / 7 / 3 / 1$ \\
\hline No. of inpatient chemotherapy, mean \pm SD (range) & $15 \pm 8(4-33)$ & $14 \pm 8(4-33)$ & $15 \pm 7(5-27)$ \\
\hline No. of outpatient chemotherapy, mean \pm SD (range) & $32 \pm 20(5-92)$ & $31 \pm 20(6-92)$ & $34 \pm 20(5-85)$ \\
\hline Objective tumor response rate & $96 \%$ & $100 \%$ & $92 \%$ \\
\hline $\begin{array}{l}\text { Inflammation-related symptoms, } \\
\text { Yes /No; No. of patients }(\%)\end{array}$ & $28(51 \%) / 27(49 \%)$ & $11(35 \%) / 20(65 \%)$ & $17(71 \%) / 7(29 \%)$ \\
\hline Death/Alive/Lost, No. of patients (\%) & $29(53 \%) / 24(44 \%) / 2(4 \%)$ & $13(42 \%) / 17(55 \%) / 1(3 \%)$ & $16(67 \%) / 7(29 \%) / 1(4 \%)$ \\
\hline $\begin{array}{l}\text { Cause of death: consc. distur./resp. } \\
\text { fail., No. of patients }(\%)\end{array}$ & $26(90 \%) / 3(10 \%)$ & $11(85 \%) / 2(15 \%)$ & $15(94 \%) / 1(6 \%)$ \\
\hline
\end{tabular}

LN: Lymph node; SD: standard deviation; cons. distur.: consciousness disturbance; resp. fail.: respiratory failure.

following the clinical treatment were observed in $82(59 \%)$ patients, including 43 (49\%) with bladder and 39 (76\%) with upper urinary tract cancers. Thromboembolism complications in all patients involved cerebral thrombosis $(n=79)$ and PVOS $(n=27)$, while paraneoplastic syndromes involved cancer cachexia $(n=44)$ and neoplastic fever $(n=3)$.

The follow-up period ranged from 2 to 126 months. Onehundred and seven patients were followed until death, with eight lost to follow-up. At the time of writing this report, 24 patients were still alive. The median survival time was 17 months (range: 2-126 months). Their 2-year, 3-year, and 5year survival rates were $41 \%, 26 \%$, and $18 \%$, respectively. The median survival time was 18 months for the bladder cancer group versus 17 months for the upper urinary tract cancer group, which was not statistically different $(p=0.5$ $\log$-rank test, Figure 1). Of the mortalities, death resulted from loss of consciousness $(n=82)$ and respiratory failure $(n=24)$.

Patients with survival time of more than two years. For the analysis of the contributory factors to the 55 patients with a survival time of more than two years, their clinical characteristics are shown in Table II. The patients included 38 men and 17 women (median age:66 years, range:40-85 years) with bladder $(n=31)$ and upper urinary tract $(n=24)$ cancers, including renal pelvis $(n=15)$ and ureter $(n=9)$. 


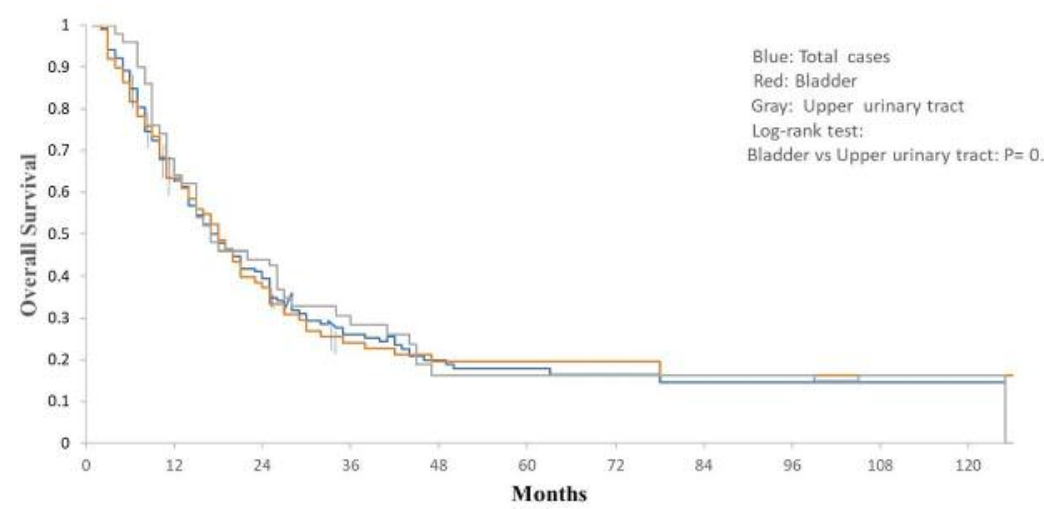

Figure 1. Survival curve of all metastatic urothelial carcinoma patients and those in bladder and upper urinary tract cancer groups. Log-rank test comparing bladder and upper urinary tract cancers.

Before chemotherapy, 35 patients had peritoneal involvement only, 7 had peritoneal involvement and lung metastases, 9 had peritoneal involvement and LN metastases, 3 had peritoneal involvement and bone metastases, and one had bone metastases only. All lung and bone metastases were oligo-metastases.

The mean number of inpatient chemotherapy cycles was $15 \pm 8$ (range: $4-33$ cycles) and the mean number outpatient chemotherapy cycles was 32 20 (range: 5-92 cycles) for all 55 patients. The overall objective response rate in all 55 patients was $96 \%$, including $100 \%$ with bladder and $92 \%$ with upper urinary tract cancers. The occurrence of inflammation-related symptoms following clinical treatment was observed in $28(51 \%)$ patients, including 11 (35\%) with bladder and $17(71 \%)$ with upper urinary tract cancers. Thromboembolism complications in all 55 patients involved cerebral thrombosis $(n=11)$ and PVOS $(n=3)$. Paraneoplastic syndromes in patients involved cancer cachexia $(n=9)$ and neoplastic fever $(\mathrm{n}=1)$.

Twenty-nine of 55 patients whose survival time was more than two years died, two were lost to follow-up, and 24 were still alive at the time of writing the manuscript. The causes of death were loss of consciousness $(n=26)$ and respiratory failure $(n=3)$.

Seven of the 24 surviving patients, including six with bladder and one with upper urinary tract cancers, continued receiving maintenance chemotherapy, two of which also had cancer cachexia under progesterone therapy. Seventeen surviving patients, including 11 with bladder and 6 with upper urinary tract cancers, stopped chemotherapy. Among them, one had cancer cachexia under progesterone therapy.

Illustrated cases with images. Figure 2 shows a bladder cancer after radical cystectomy and concomitant CCRT, but the tumor progressed with peritoneal spreading and abdominal wall
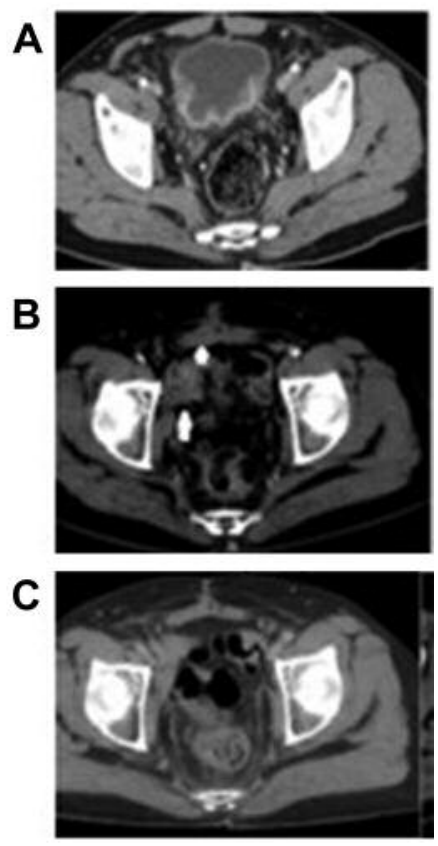

Figure 2. A 56-year-old male metastatic urothelial carcinoma patient with bladder cancer with peritoneal spreading and abdominal wall invasion, who survived for more than two years. (A) CT scan showing a bladder malignancy. The patient received radical cystectomy and then concomitant chemoradiotherapy, but the tumor progressed. (B) CT scan showing pelvis peritoneal thickening and adjacent abdominal wall invasion. Continuous maintenance chemotherapy was administered. (C) CT scan at a 3-year follow-up showing tumor regression with fibrosis and healing. He survived for more than 4.5 years. Arrows indicate thickening of the peritoneum and adjacent abdominal wall invasion.

invasion. The patient received continuous maintenance chemotherapy and the tumor responded with fibrosis and healing. 
A

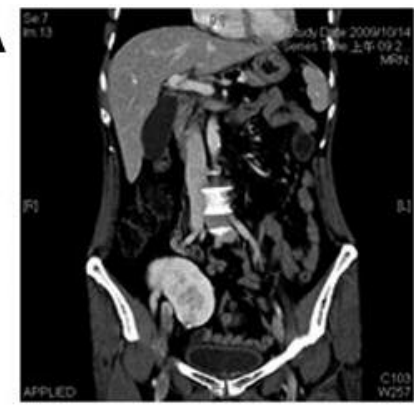

B

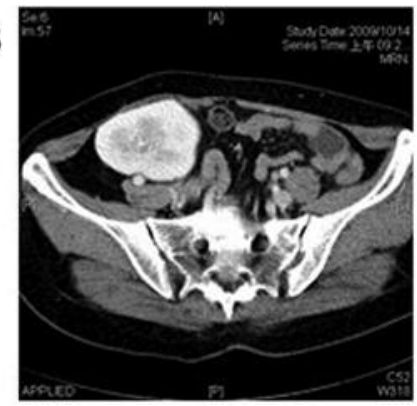

C

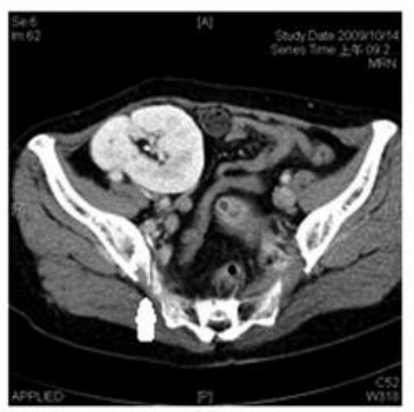

$\mathbf{E}$

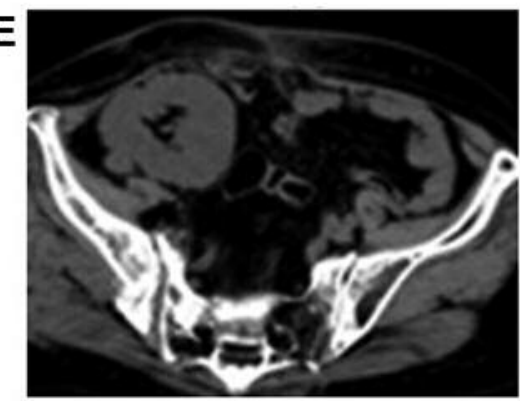

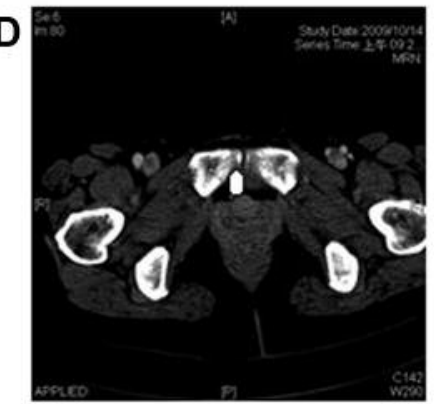

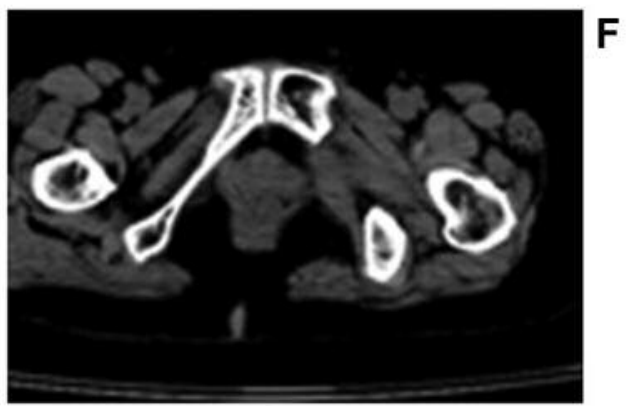

Figure 3. A 44-year-old female metastatic urothelial carcinoma patient with right middle ureteral cancer with peritoneal spreading and bony oligometastases post renal transplantation, who survived for more than two years. CT scans showing (A) a right middle malignancy, (B) peritoneal thickening and adjacent sacral vertebral body invasion, $(C)$ right ischial bony metastasis, and (D) pubic bony metastasis. Following nephronureterectomy, she received continuous maintenance chemotherapy, resulting in tumor regression. CT scan at the 8-year follow-up showed (E) right ischial bone and $(F)$ pubic bone fibrosis and healing. She survived for more than 8.5 years and without chemotherapy for two years. Arrow indicates thickening of the peritoneum. Arrowheads indicate bony invasion or metastasis.

Figure 3 shows a right middle ureteral cancer with peritoneal spreading and bony oligo-metastases post renal transplant. Following nephron-urecteromy, the patient received continuous maintenance chemotherapy, whereby the tumor regressed with pubic bone fibrosis and healing.

Figure 4 shows a bladder cancer following radical cystoprostatectomy with peritoneal spreading and abdominal wall invasion. The patient received continuous maintenance chemotherapy. The tumor responded with fibrosis and healing, but regularly changing his Double $\mathrm{J}$ stent accompanied by frequent urinary tract infections bothered him. The tumor recurred over the perineum with cachexia and coagulopathy and the patient eventually died.
Figure 5 shows a left upper ureteral cancer with peritoneal spreading and lung oligo-metastases. The patient presented with dyspnea and cachexia and received continuous maintenance chemotherapy and progesterone therapy. The tumor regressed and chemotherapy was ceased for more than four years, but the lung tumor recurred with cachexia and coagulopathy.

Figure 6 shows a left renal pelvis cancer with peritoneal spreading, bony oligo-metastases, and lung metastases. The patient received continuous maintenance chemotherapy, whereby the tumor regressed but recurred with coagulopathy.

Figure 7 shows a bladder cancer with peritoneal spreading. The patient received continuous maintenance 

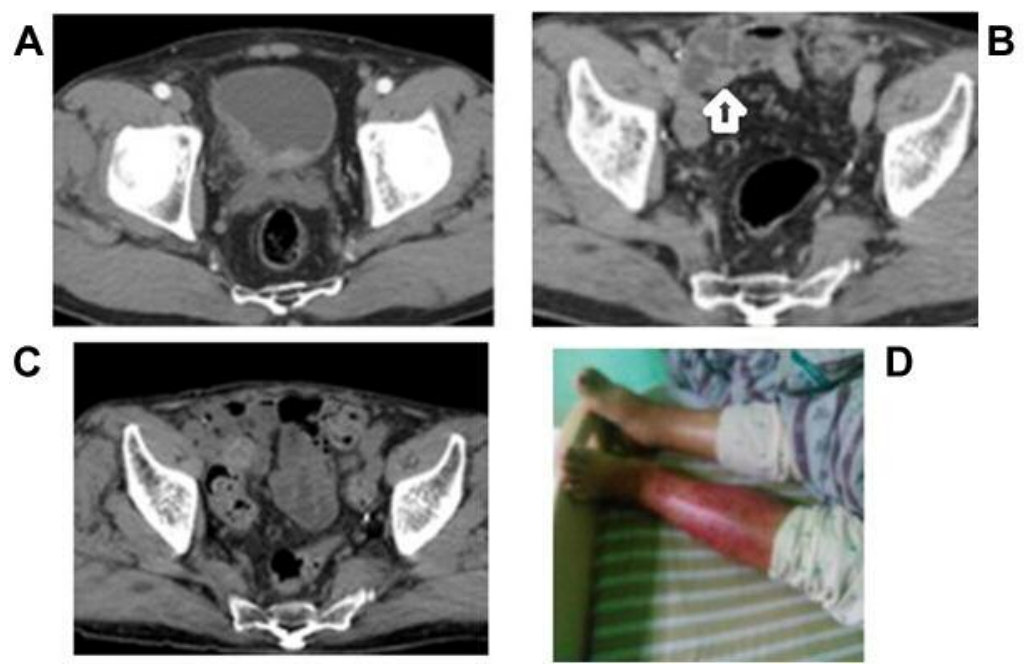

Figure 4. A 66-year-old male metastatic urothelial carcinoma patient with bladder cancer with peritoneal spreading and abdominal wall invasion, who survived for more than two years. (A) CT scan showing a bladder malignancy with prostate invasion. Following radical cysto-prostatectomy, he received continuous maintenance chemotherapy. (B) CT scan showing pelvis peritoneal thickening and adjacent abdominal wall invasion. (C) $C T$ scan at the 3.5-year follow-up showed tumor response with fibrosis and healing. However, regularly changing the D-J stent with frequent urinary tract infection bothered him. He discontinued chemotherapy for two years. Eventually tumors recurred at the perineum with cachexia and coagulopathy. (D) Photo showing lower left leg thrombophlebitis. After surviving for five years, he died. Arrow indicates thickening of the peritoneum and adjacent abdominal wall invasion.

chemotherapy for 5.5 years and the tumor responded completely. Chemotherapy was discontinued for 2.5 years, but the bladder cancer with peritoneal spreading recurred over the original area.

Figure 8 shows a right renal pelvis cancer with bony oligo-metastases and cancer cachexia. The patient received nephro-ureterectomy with continuous maintenance chemotherapy and progesterone therapy.

Figure 9 shows a left upper ureteral cancer with a L3 vertebral body invasion. The patient received continuous maintenance chemotherapy, which resulted in tumor regression. However, nephro-ureterectomy was performed later and the tumor rapidly progressed with sacral metastasis.

Figure 10 shows a bladder cancer with peritoneal spreading, abdominal wall invasion, and right inguinal hernia formation. The patient received continuous maintenance chemotherapy. However, following hernia repair surgery, the tumor rapidly progressed with cancer cachexia.

Figure 11 shows a left lower ureteral cancer with peritoneal spreading. The patient received nephro-ureterectomy and then continuous maintenance chemotherapy. The tumor regressed but showed left lung metastatic lesion. The patient received metastectomy and continuous chemotherapy.

Figure 12 shows a left middle ureteral cancer with peritoneal spreading. The patient received a nephro-ureterectomy and then continuous maintenance chemotherapy. A right lower lung metastatic lesion appeared and the tumor invaded into the proximal gastrointestinal tract with bleeding and iliac vein narrowing due to obstruction of the left iliofemoral vein with left lower extremity vasogenic and lymphatic edema. Here, the tumor regressed following chemotherapy.

Contributing factors. As seen in the clinical images, all 11 patients demonstrated an objective response with no unfavorable metastatic conditions. All patients exhibited various healing processes with fibrosis formation (Figures 24, and 12).

Three patients had symptoms related to inflammation including cachexia and coagulopathy (Figures 4-6, and 10). Five patients (bladder cancer in 4 and upper urinary tract in one) underwent surgery, with rapid tumor progression noted (Figures 9 and 10). Two patients with upper urinary tract cancer (one lower ureteral cancer and another renal pelvis cancer combined with localized bladder cancer) received an invasive procedure (cystoscope with biopsy) but saw rapid tumor progression. One patient with renal pelvis cancer relapse had liver metastases and rapid tumor progression after incomplete radiofrequency ablation.

One bladder cancer patient with peritoneal spread and abdominal wall invasion frequently experienced urinary tract infections. Eventually, regional tumor recurrence and inflammation-related symptoms occurred (Figure 4).

Two bladder cancer cases had later recurrence following chemotherapy (Figure 7). One bladder cancer with peritoneal 


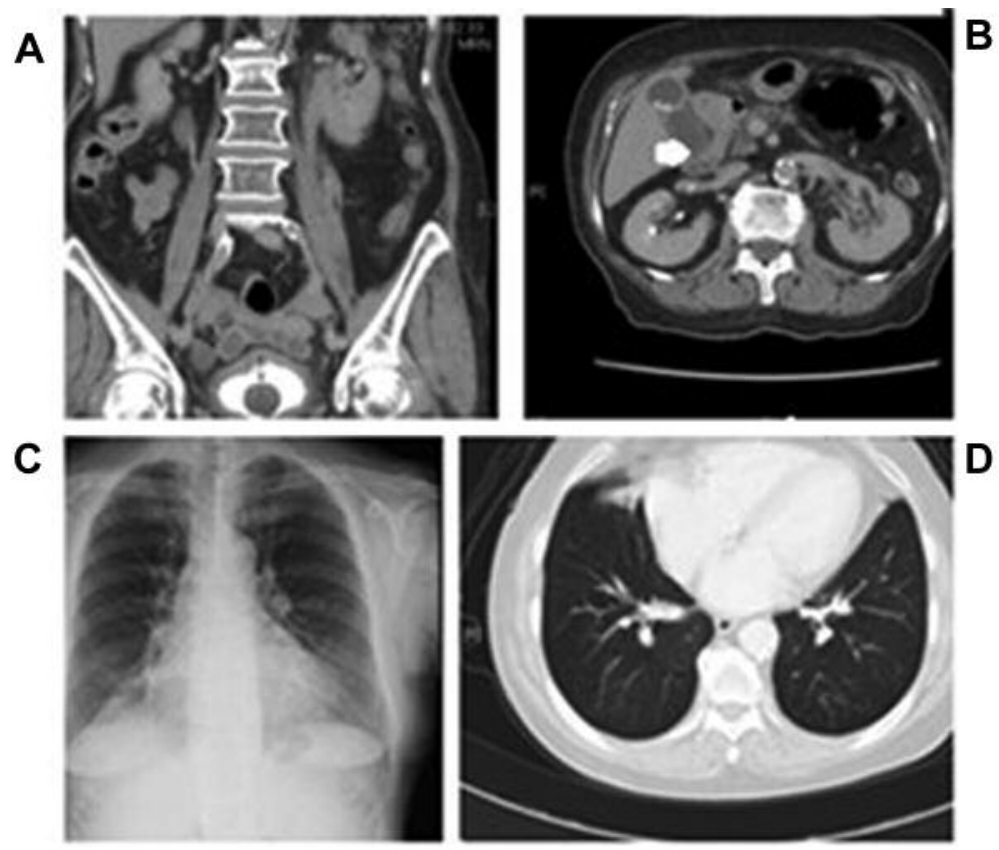

Figure 5. A 74-year-old female metastatic urothelial carcinoma patient with left upper ureteral cancer with peritoneal spreading and lung oligometastases, who survived for more than two years. $(A, B) C T$ scans showing a left upper ureteral malignancy with left hydroureter. She presented with dyspnea and cachexia. She received continuous maintenance chemotherapy and progesterone agent therapy. (C) Chest X-ray and (D) CT scan showed right medial lung infiltration. The tumor regressed and chemotherapy was stopped for four years and four months; however, the right lung lesions recurred with cachexia and coagulopathy. After surviving for six years and eight months, she died. Arrows indicate thickening of the peritoneum.
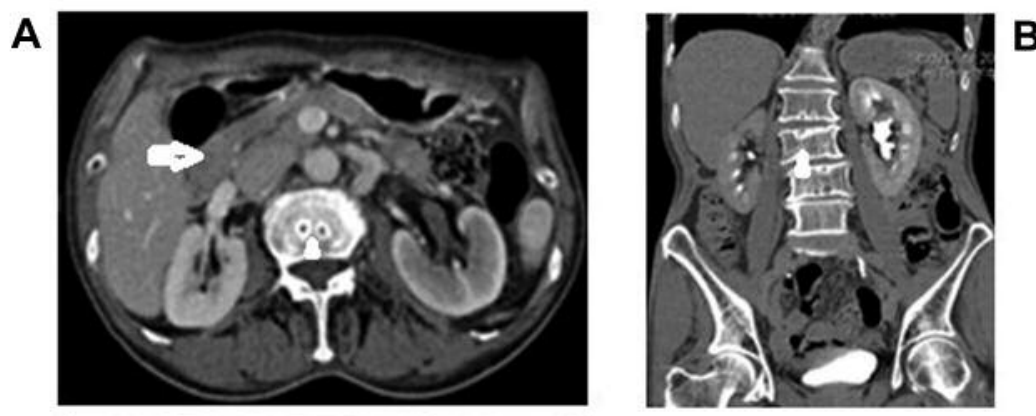

C
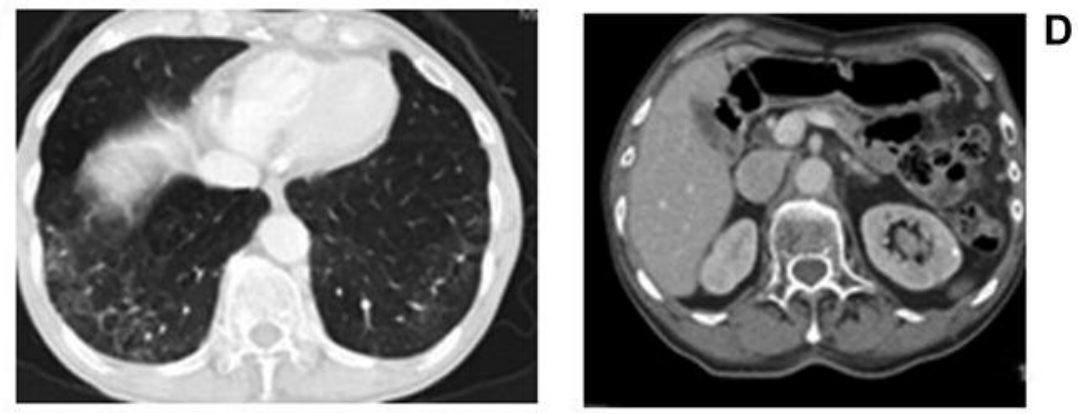

Figure 6. An 80-year-old male patient with metastatic urothelial carcinoma undergoing continuous maintenance chemotherapy for left renal pelvis cancer with peritoneal spreading, bony oligo-metastases, and lung metastases, and survived for more than two years. (A, B) CT scans showing a left renal pelvis cancer with peritoneal thickening, bony metastases. (C) CT scan showing lung infiltrating lesions two years following chemotherapy. (D) CT scan showing decreased peritoneal thickening and bone lesion regression. His tumors recurred with coagulopathy, and he died after surviving for four years and eight months. Arrow indicates thickening of the peritoneum. Arrowheads indicate vertebral metastases. 

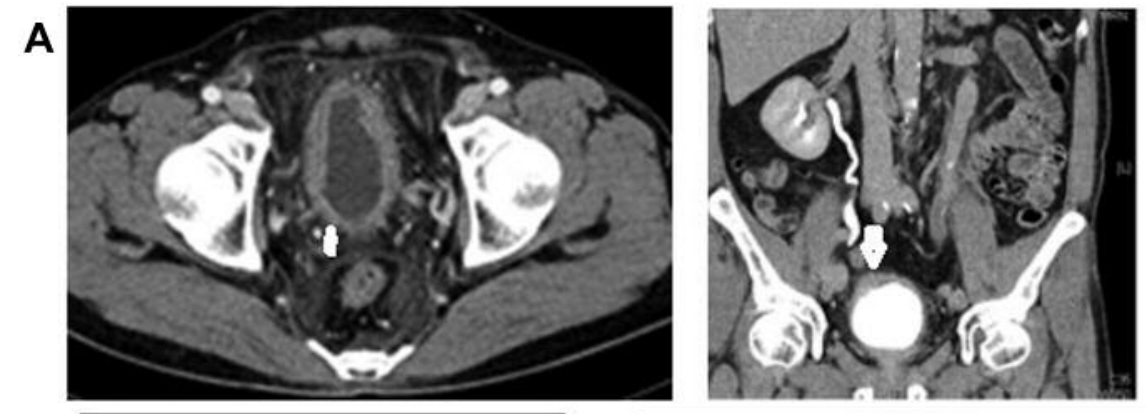

C
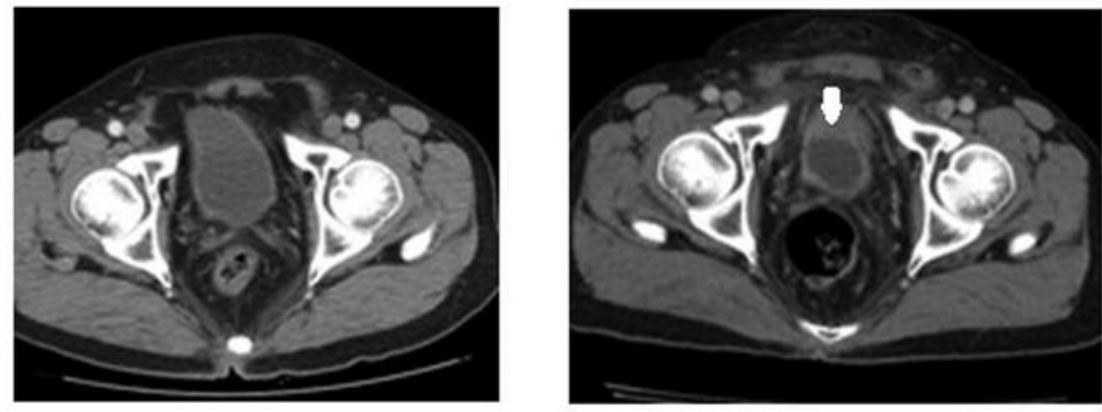

B

D

Figure 7. A 66-year-old male metastatic urothelial carcinoma patient with bladder cancer with peritoneal spreading, who survived for more than two years. The patient received continuous maintenance chemotherapy. (A, B) CT scans showing bladder cancer with peritoneal spreading. (C) CT scan at the 5.5-year follow-up showing tumor regression. Chemotherapy was discontinued for 2.5 years. (D) CT scan showing recurring bladder cancer with peritoneal spreading over original area. He survived more than 7.5 years. Arrows indicate peritoneal spreading.

A
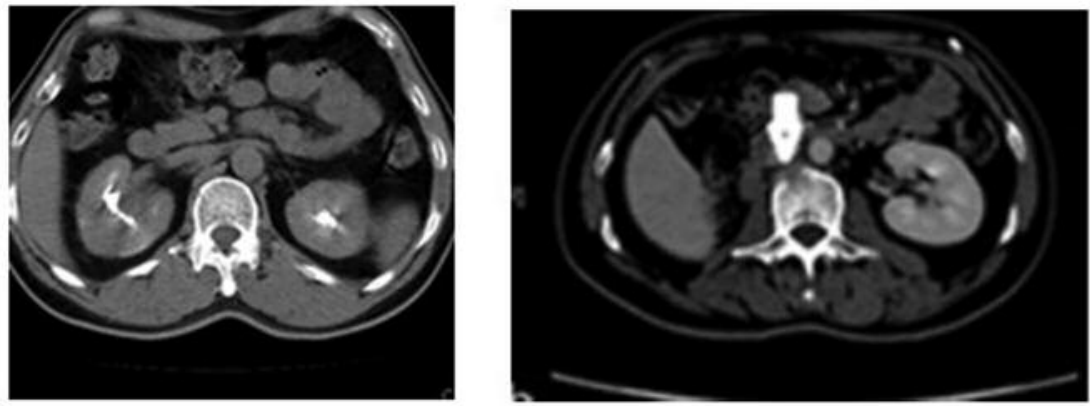

B
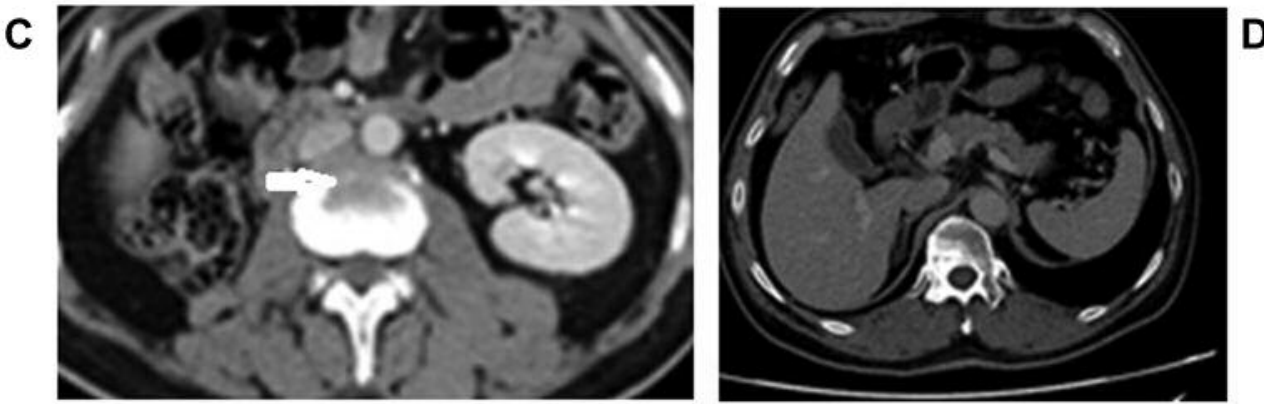

Figure 8. A 57-year-old male metastatic urothelial carcinoma patient with right renal pelvis cancer with bony oligo-metastases, who survived for more than two years. The patient received nephron-ureterectomy. (A) CT scan showing a right renal pelvis cancer. After surgery, he presented with radicular pain and cachexia. $(B, C) C T$ scans showing lumbar vertebral body osteolytic lesion for which he received continuous maintenance chemotherapy and progesterone agent therapy. (D) CT scan at the 3-year follow-up showed sclerotic bone lesions. He survived for more than 4.5 years. Arrows indicate vertebral body osteolytic lesions. 

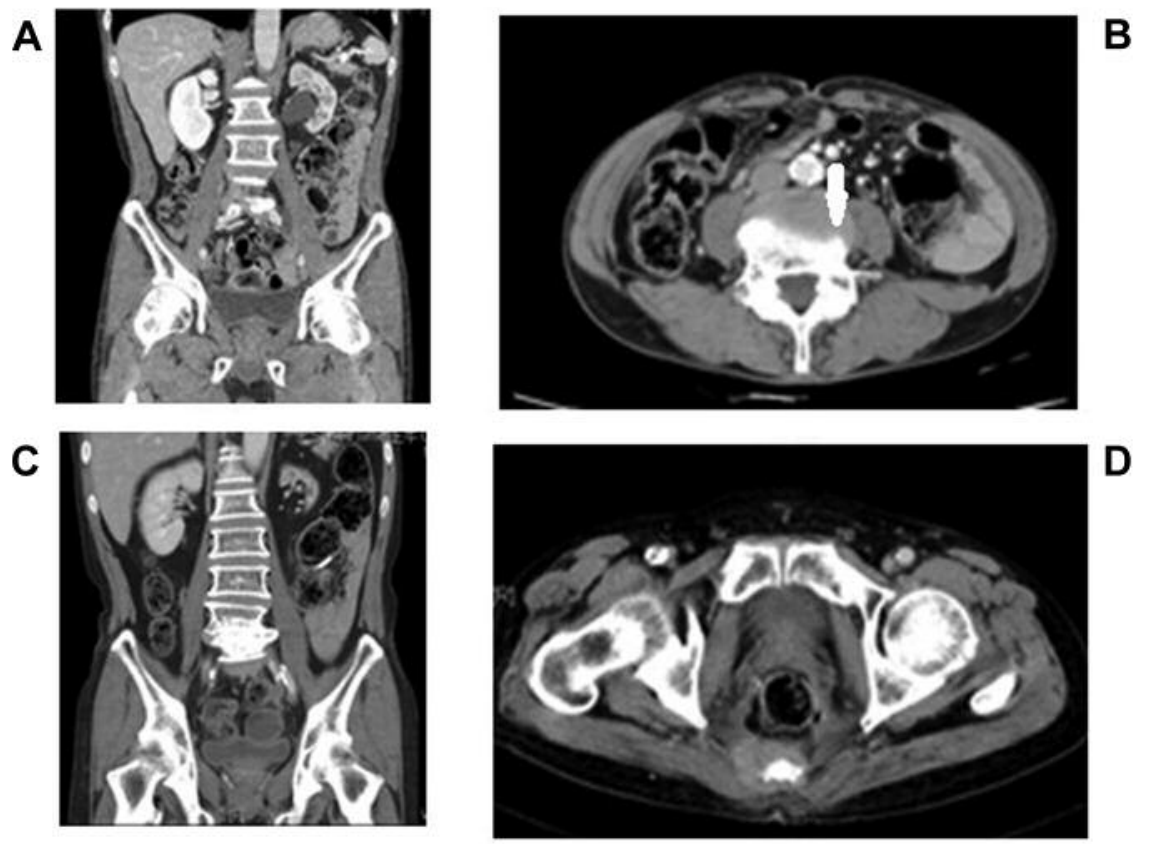

Figure 9. A 65-year-old male metastatic urothelial carcinoma patient with left upper ureteral cancer with L3 vertebral body invasion, who survived for more than two years. The patient received continuous maintenance chemotherapy. $(A, B) C T$ scans showing a left upper ureteral cancer with $L 3$ vertebral body invasion. (C) CT scan at the 18-month follow-up showing tumor regression. He received a nephron-ureterectomy. (D) CT scan showing rapid tumor progression with sacral metastasis. After surviving for two years and one month, he died. Arrows indicate vertebral body destruction lesions.

spreading and right iliac, paraaortic, and left supraclavicular LN metastases received continuous maintenance chemotherapy for 2.5 years; nine years later, tumor recurrence was observed at the original area.

The tumor in a renal pelvis cancer patient with peritoneal spreading and lung oligo-metastases with cancer cachexia completely regressed following therapy (Figure 5). Four cases, including three with upper urinary tract and one with bladder cancer, had an episode of lung metastases during chemotherapy (Figure 12). One ureteral cancer patient had lung metastases during the course of chemotherapy and underwent lung metastectomy (Figure 1).

One patient with ureteral cancer also developed salivary and bladder cancer after being disease-free (Figure 11). Another bladder cancer occurred with acute myeloid leukemia after tumor regression following chemotherapy. Thirteen patients, including eight with bladder and five with upper urinary tract cancers, were potentially cured (Figures 3 and 12).

\section{Discussion}

Survival time for metastatic urothelial carcinoma. The median survival time for our MUC patients receiving continuous maintenance chemotherapy was 17 months, with no difference between bladder and upper urinary tract cancer location (18 versus 17 months). The survival time was compatible with previous reports $(1,13,14)$. Patient survival was likely related not only to the objective tumor response, but also to inflammation and coagulopathy as major contributory factors to patient death, as demonstrated by the inflammation-related symptoms and cause of death.

Concept of continuous maintenance chemotherapy. The objective tumor response rate of our MUC patients receiving continuous maintenance chemotherapy was $60 \%$. In our previous report, bladder urothelial carcinomas patients with peritoneal spreading benefited from continuous maintenance chemotherapy with an overall response rate of $85 \%$ and median survival time of 28 months (2). A Cisplatin dose of $50 \mathrm{mg} / \mathrm{m}^{2}$ can allow for more doses of the drug and prevent toxicity. Initially aggressive therapy led to tumor response, and the administration of chemotherapy was slowed down when the condition improved. The latter is a metronomic concept (i.e., reduced doses given at regular and frequent time intervals). Tumors often overcome the cytotoxic effects of chemotherapy and their microenvironment may facilitate progression and metastatic dissemination (15). Metronomic chemotherapy can involve both the tumor and its microenvironment, thereby minimizing therapeutic resistance $(16,17)$. 
A
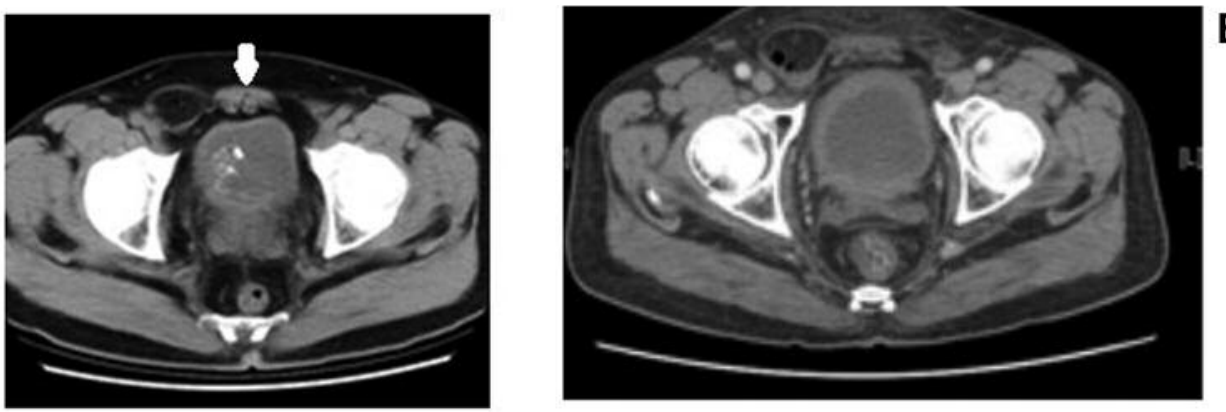

B
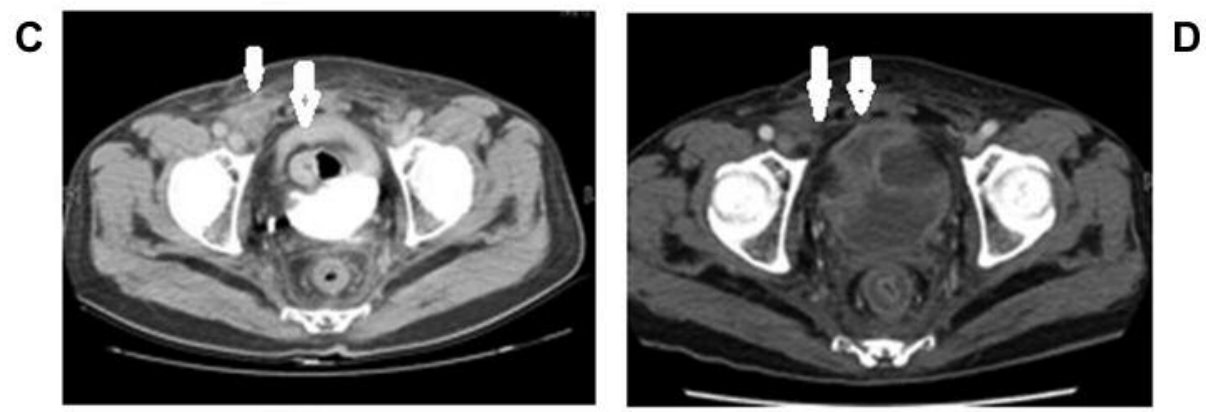

Figure 10. A 60-year-old male metastatic urothelial carcinoma patient with bladder cancer with peritoneal spreading, abdominal wall invasion, and right inguinal hernia formation, who survived for more than two years. (A) CT scan showing a bladder malignancy and right inguinal hernia formation for which he received continuous maintenance chemotherapy. (B) CT scan at the 1-year follow-up showing tumor response. He then underwent hernia repair surgery. Three months later, the tumor rapidly progressed. (C) CT scan showing pelvis peritoneal thickening and adjacent abdominal wall invasion. (D) Six months later, the tumor remained unresponsive to chemotherapy. After surviving for four years, he died. Arrows indicate thickening of the peritoneum and adjacent abdominal wall invasion.

Concept of treatment. Strategy of survival benefit for MUC patients receiving continuous maintenance chemotherapy are listed in Figure 13. The strategy of treatment includes: i) tumors responding to therapy, ii) the utilization of wound healing, and iii) the prevention of inflammation.

Continuous maintenance chemotherapy using the metronomic concept was targeted to chemo-sensitive tumors without unfavorable metastases and their response to chemotherapy was observed. The utility of the healing process led to fibrosis formation after the tumor responded to treatment, however, cancer cells remained within the stroma. Prevention of inflammation in this context can include decreasing cancer cytokine production and prevention of cytokine production by the host in reaction to surgery, invasive procedures, or infections $(3,4)$.

Instances without an unfavorable metastatic condition. Peritoneal involvement due to MUC is commonly seen. While such lesions are often overlooked, under-staged, and difficult to confirm $(18,19)$, they responded to chemotherapy in our previous report (2).

In the current study, nine patients with LN metastases had a survival time of more than two years. MUC with LN metastases only was reported to not be an adverse factor like other site metastases (20). In addition, seven patients with lung oligo-metastases had a survival time of more than two years. Temporal episodes of lung metastases can occur during the course of chemotherapy. Lung metastasectomy was feasible in one patient. Long-term survival has been reported for some patients with MUC who underwent metastasectomy (21). Four patients with bone oligometastases had a survival time of more than two years. Bone metastases carries a poor prognosis (22), but long-term survival in bone-predominant metastatic urothelial carcinoma has been reported (23).

The poor prognostic outcome associated with multiple lung metastases from MUC can be explained by drug resistance. Metastatic lesions enter the lung parenchyma from the pulmonary veins and tumor resistance can develop during the EMT period $(4,24)$. TGF- $\beta$ can also promote heterogeneity and drug resistance from the action of cancer stem cells and stromal cells in the tumor microenvironment (25). Multiple bone metastases from metastatic urothelial carcinoma have been reported with poor prognosis (2).

Utility of healing process and fibrosis formation. After tumors respond to chemotherapy, they undergo a wound healing process, such as following acute injury. Rybinski et 
A
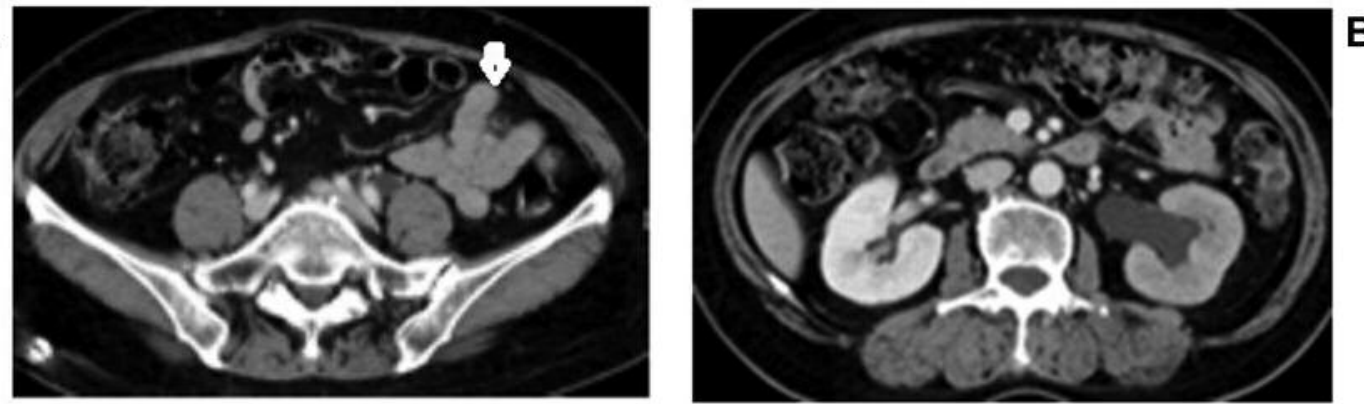

B
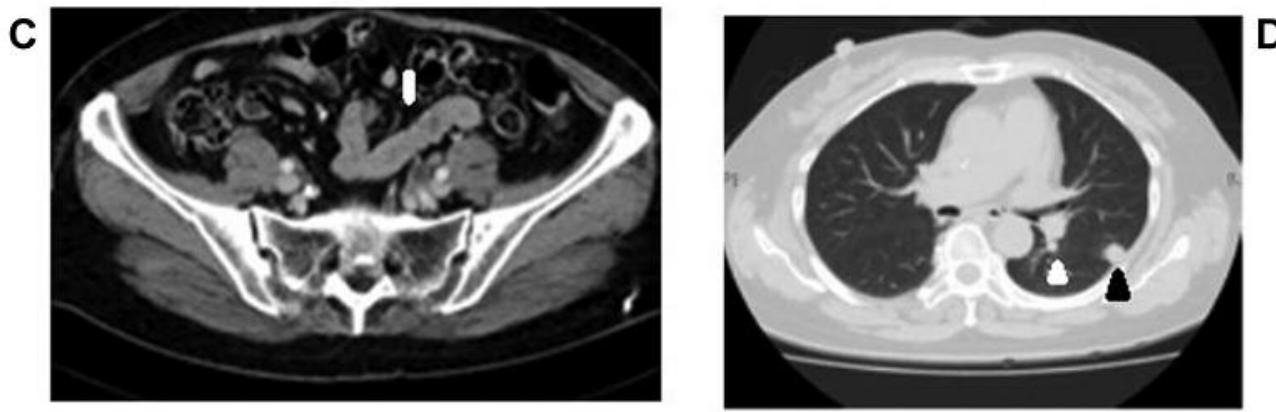

Figure 11. A 66-year-old female metastatic urothelial carcinoma patient with lower left ureteral cancer with peritoneal spreading, who survived for more than two years. (A) CT scan showing a lower left ureteral cancer. Following nephron-ureterectomy, she received continuous maintenance chemotherapy. (A-C) CT scans showing a lower left ureteral cancer and renal pelvis cancer with peritoneal thickening. (D) The tumors regressed, and one year and 10 months later, CT scans showed left lung metastatic lesions. She received metastectomy and continued maintenance chemotherapy. She discontinued chemotherapy for one year. She died of secondary salivary gland malignancy after surviving for 10.5 years. Arrow indicates thickening of the peritoneum. Arrowheads indicate lung metastases.

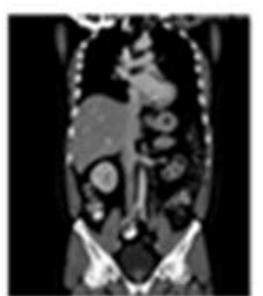

A

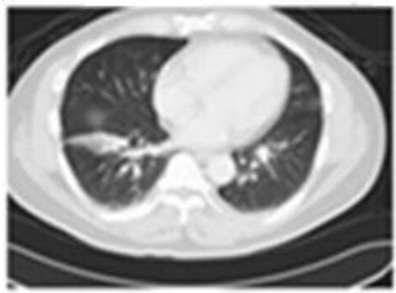

E

B
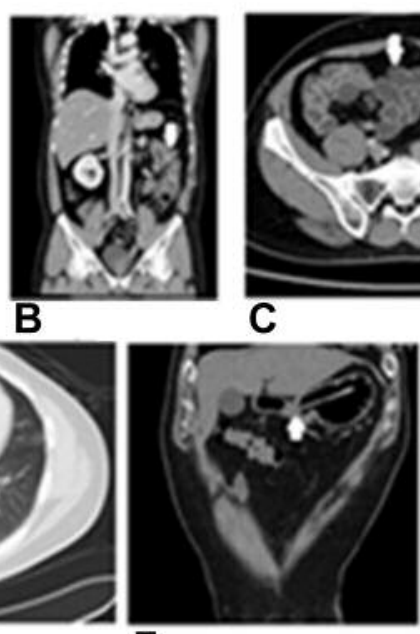

F

C
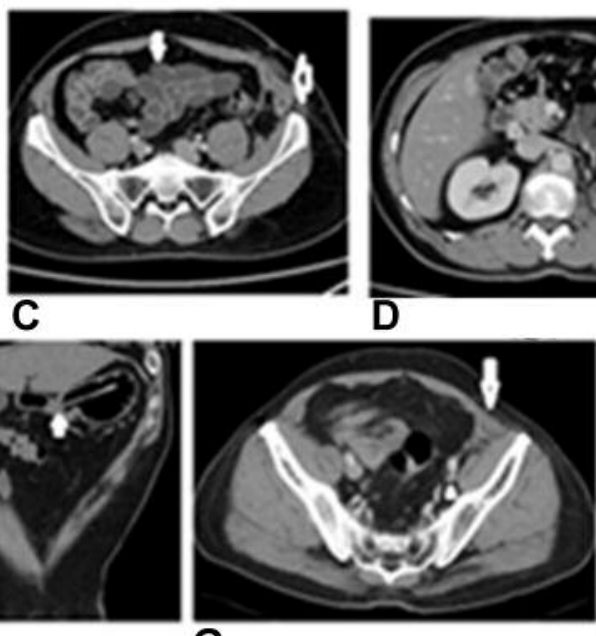

G

D
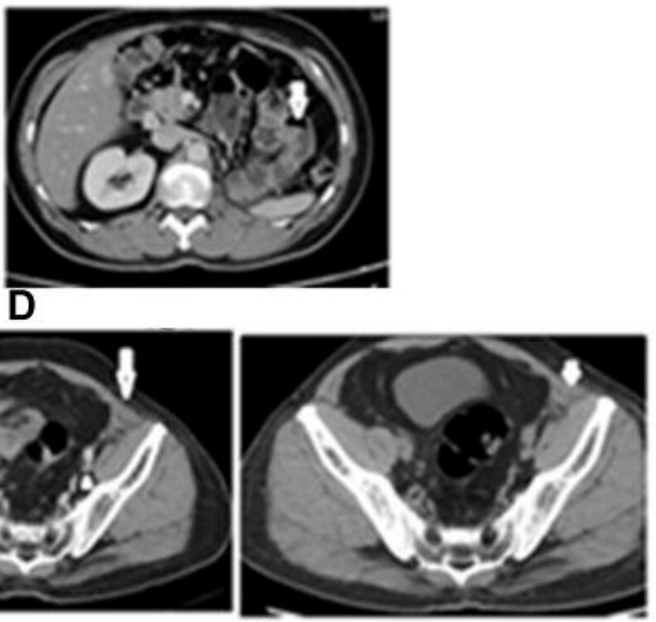

H

Figure 12. A 53-year-old male metastatic urothelial carcinoma patient with left middle ureteral cancer with peritoneal spreading, who survived for more than two years. After nephron-ureterectomy, the patient received continuous maintenance chemotherapy. (A) CT scan showing a left middle ureteral cancer. (B-D) After nephron-ureterectomy, $C T$ scans show a 'dirty; tumor with peritoneal thickening. (E) CT scan showing a right lower lung metastatic lesion. $(F)$ CT scan showing tumor invasion into proximal gastrointestinal tract with bleeding. $(G) C T$ scan showing iliac vein narrowing due to left iliofemoral vein obstruction with left lower extremity vasogenic and lymphatic edema. He continued chemotherapy. (H) CT scan showing tumor regression. He discontinued chemotherapy for 18 months and survived for 5.5 years. 
al., have reported that the process of wound healing displays similar characteristics to chronic fibrosis (27). The healing process during acute injury involves four components: i) coagulation, ii) inflammation, iii) cellular proliferation, and iv) extracellular matrix (ECM) repair (27). Normal fibroblasts can prevent the initiation and metastases of cancer cells via direct cell-cell contact, paracrine signaling by soluble factors, and ECM integrity. However, cancer cells still reside within the stroma (28). Cancer-associated fibroblasts (CAFs) can release regulatory factors into the tumor microenvironment that have essential roles, including the support of tumor growth, angiogenesis, metastasis, and resistance to therapy (29). Furthermore, CAFs produce cytokines that promote the biological characteristics of tumors $(29,30)$. In our patients, tumors recurred at the original location in spite of continuous maintenance therapy or cessation of therapy for several years. Some patients continue to experience inflammatory activity, such as cancer cachexia, but such diseases can be controlled.

Decrease in tumor-associated inflammation. Solid tumors consist of neoplastic cells, non-malignant stromal cells, and migratory hematopoietic cells (31). Tumor associated macrophages (TAMs) are key players in the link between inflammation and cancer (31). Complex interactions in this microenvironment regulate tumor growth, progression, metastasis, and angiogenesis (31-33). Targeting these inflammatory responses may improve patient outcomes (34). Clinicians use dexamethasone and low molecular weight heparin for PVOS and cerebral thrombosis, progesterone agents for cancer cachexia, and naproxen for neoplastic fever $(3,4,8,9)$. These therapies all suppress cytokine production.

Prevention of inflammatory host reactions. Tumor stroma generation and wound healing share many common features, including immune response, cell proliferation, cell migration, tissue remodeling, and cell death (35). Standard therapies aimed at fibrosis formation due to wound healing can prevent tumor growth (36). Surgery may destroy repaired tumor tissue and let cancer cells spread into the stroma. CAFs also play a role in cytokine production that promotes tumor progression. In our patients receiving inguinal hernia repair surgery and nephron-ureterectomy, tumor repair tissue was damaged and cytokine production increased. This surgical stress resulted in rapid tumor progression (37). One case receiving incomplete radiofrequency ablation for metastatic liver tumors had enhanced invasiveness and metastasis of the residual cancer (38). Surgical procedures, such as tumor resection and biopsy are still standard for the diagnosis and treatment of solid tumors; however, even minor surgical trauma can promote postoperative tumor spreading (39). Two of our cases received cystoscopy with biopsy and rapid tumor progression occurred. Chronic
Tumor response 1. Chemo-sensitive tumor

2. No multiple visceral and

bone metastatic site

3. Metronomic concept

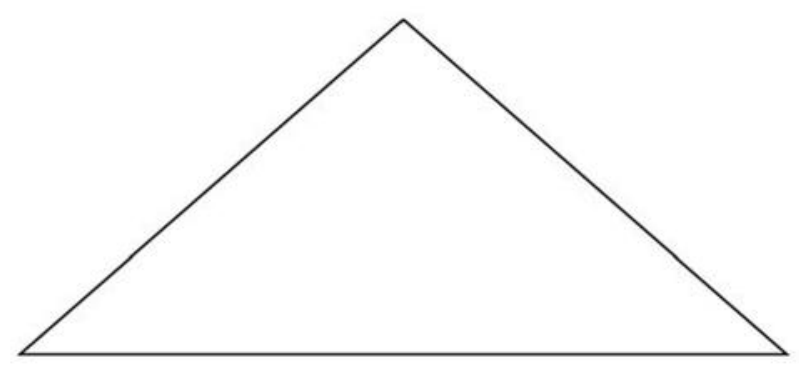

Produce fibrosis

1. Tumor response $\rightarrow$ good healing and fibrosis formation, but cancer cells remain within the stroma
Decrease inflammation

1. Decrease tumor cytokines

2. Prevent host reaction induce cytokines production (such as in response to surgery, invasive procedure, or infection)
Figure 13. Strategy of survival benefit for metastatic urothelial carcinoma patients receiving continuous maintenance chemotherapy, including responsive tumor with metronomic concept, producing fibrosis and decrease production of inflammation.

inflammation can influence the tumor microenvironment and allow nascent tumors to escape immunosurveillance $(40,41)$.

Secondary malignancy. Acute myeloid leukemia, similar to our patients' condition, can occur following platinum-based chemotherapy (42). However, secondary malignant salivary gland tumors, unlikely in our patients, occurred only in survivors of childhood cancer (43).

Limitations. Our study has several important limitations. First, the data was collected from a retrospective case cohort study in a single center and mainly from a single physician. Second, peritoneal thickening diagnoses were based on CT scans only and were not confirmed by pathology. Third, no cytokine studies were performed. Fourth, consciousness/ mental changes related to thromboembolic complication are seldom proven by image studies. Fifth, the diagnoses were not confirmed by tissue samples (either by biopsy or autopsy).

Continuous maintenance chemotherapy for chemosensitive tumors, applied via a metronomic schedule and focus on tumor microenvironment, can lead to survival benefits for patients with MUC. The wound healing process can prevent tumor spreading but not fully eradicate cancer. Decreased inflammation related to tumor and host reactions can also prevent tumor growth. 


\section{Conflicts of Interest}

The Authors declare no conflicts of interest.

\section{Authors' Contributions}

CCL was responsible for the concept of the study, data analysis and writing/editing of the manuscript. TYL, KHT and YHJ were responsible for the concept of the study and data analysis. All authors read and approved the final version of the manuscript.

\section{Acknowledgements}

The Authors wish to acknowledge the oncology nursing staff for providing the best care for these patients. They would like also to thank Uni-edit (www.uni-edit.net) for editing and proofreading this manuscript.

\section{References}

1 Matsumoto R, Abe T, Ishizaki J, Kikuchi H, Harabayashi T, Minami K, Sazawa A, Mochizuki T, Akino T, Murakumo M, Osawa T, Maruyama S, Murai S and Shinohara N: Outcome and prognostic factors in metastatic urothelial carcinoma patients receiving second-line chemotherapy: an analysis of real-world clinical practice data in Japan. Jpn J Clin Oncol 48: 771-776, 2018. PMID: 29939285. DOI: 10.1093/jjco/hyy094

2 Liaw CC, Chuang CK, Chang YH, Liao TY, Chang JW and Juan YH: Bladder urothelial carcinoma with peritoneal involvement: benefit of continuous maintenance chemotherapy. Anticancer Res 37: 6443-6451, 2017. PMID: 29061831. DOI: 10.21873/ anticanres.12099

3 Liaw CC, Chang H, Yang TS and Wen MS: Pulmonary venous obstruction in cancer patients. J Oncol 2015: 210916. 2015. PMID: 27746816. DOI: 10.1155/2016/1872627

4 Liaw CC, Chang H, Liao TY, Wen MS, Yu CT and Juan YH: The role of pulmonary veins in cancer progression from a computed tomography viewpoint. J Oncol 2016: 1872627, 2016. PMID: 27746816. DOI: 10.1155/2016/1872627

5 Papageorgis P: TGF $\beta$ signaling in tumor initiation, epithelial-tomesenchymal transition, and metastasis. J Oncol 2015: 587193, 2015. PMID: 25883652. DOI: 10.1155/2015/587193

6 Fukushima H, Yokoyama M, Nakanishi Y, Tobisu K and Koga F: Sarcopenia as a prognostic biomarker of advanced urothelial carcinoma. PLoS One 10: e0115895, 2015. PMID: 25612215. DOI: $10.1371 /$ journal.pone.0115895

7 Jang GS, Kim MJ, Ha HI, Kim JH, Kim HS, Ju SB and Zang DY: New response evaluation criteria in solid tumors: revised RECIST guideline (version 1.1). Eur J Cancer 48: 689-694, 2013. PMID: 24385696. DOI: 10.3978/j.issn.1000-9604.2013. 11.09

8 Suzuki H, Asakawa A, Amitani H, Nakamura N and Inui A: Cancer cachexia: pathophysiology and management. J Gastroenterol 48: 574-594, 2013. PMID: 23512346. DOI: 10.1007/s00535-013-0787-0

9 Liaw CC, Huang JS, Chen JS, Chang JW, Chang HK and Liau CT: Using vital sign flow sheets can help to identify neoplastic fever and other possible causes in oncology patients: a retrospective observational study. J Pain Symptom Manage 40:
256-265, 2010. PMID: 20598848. DOI: 10.1016/j.jpainsymman. 2010.01 .015

10 Yücel B, Celasun MG, Öztoprak B, Hasbek Z, Bahar S, Kaçan T, Bahçeci A and Şeker MM: The negative prognostic impact of bone metastasis with a tumor mass. Clinics (Sao Paulo) 70: 535540, 2015. PMID: 26247664. DOI: 10.6061/clinics/2015(08)01

11 Mertens LS and Horenblas S: Bladder cancer: oligometastases and imaging. Nat Rev Urol 14: 513-514, 2017. PMID: 28631741. DOI: $10.1038 /$ nrurol.2017.96

12 Foster DS, Jones RE, Ransom RC, Longaker MT and Norton JA: The evolving relationship of wound healing and tumor stroma. JCI Insight 3 pii: 99911, 2018. PMID: 30232274. DOI: 10.1172/jci.insight.99911

13 Niegisch G, Gerullis H, Lin SW, Pavlova J, Gondos A, Rudolph A, Haas G, Hennies N and Kramer MW: A real-world data study to evaluate treatment patterns, clinical characteristics and survival outcomes for first- and second-line treatment in locally advanced and metastatic urothelial cancer patients in Germany. J Cancer 9: 1337-1348, 2018. PMID: 29721042. DOI: 10.7150/ jca.23162

14 Hsieh MC, Chiang PH, Rau KM, Chen YY, Su YL and Huang $\mathrm{CH}$ : The comparison of oncologic outcomes between metastatic upper tract urothelial carcinoma and urothelial carcinoma of the bladder after cisplatin-based chemotherapy. Urol Oncol 33: 495.e9-495.e14, 2015. PMID: 26254698. DOI: 10.1016/ j.urolonc.2015.07.001

15 Karagiannis GS, Condeelis JS and Oktay MH: Chemotherapyinduced metastasis: mecanisms and translational opportunities. Clin Exp Metastasis 35: 269-284, 2018. PMID: 29307118. DOI: 10.1007/s 10585-017-9870-x

16 Kareva I, Waxman DJ and Lakka Klement G: Metronomic chemotherapy: an attractive alternative to maximum tolerated dose therapy that can activate anti-tumor immunity and minimize therapeutic resistance. Cancer Lett 358: 100-106, 2015. PMID: 25541061. DOI: 10.1016/j.canlet.2014.12.039

17 Gasparini G: Metronomic scheduling: the future of chemotherapy? Lancet Oncol 2: 733-740, 2001. PMID: 119 02515. DOI: $10.1016 / \mathrm{S} 1470-2045(01) 00587-3$

18 Liaw CC, Chuang CK, Chang YH, Chang JW, Liao TY and Juan YH: Under-stage and overlook of peritoneal spread from bladder urothelial carcinoma. In Vivo 32: 905-910, 2018. PMID: 29936478. DOI: 10.21873/invivo.11327

19 Liao TY, Liaw CC, Tsui KH and Juan YH: Pre-therapy CT scan showing peritoneal thickening from metastatic renal pelvis carcinoma patients. Med Oncol 35: 128, 2018. PMID: 30116986. DOI: $10.1007 / \mathrm{s} 12032-018-1181-9$

20 Salah S, Lee JL, Rozzi A, Kitamura H, Matsumoto K, Srinivas S, Morales-Barrera R, Carles J, Al-Wardat R, Al-Rabi K and Maakoseh M: Second-line chemotherapy for metastatic urothelial carcinoma: importance of lymph node-only metastasis as a prognostic factor and construction of a prognostic model. Clin Genitourin Cancer 14: 255-260, 2016. PMID: 26552764. DOI: 10.1016/j.clgc.2015.10.006

21 Nakagawa T, Taguchi S, Kanatani A, Kawai T, Ikeda M, Urakami S, Matsumoto A, Komemushi Y, Miyakawa J, Yamada D, Suzuki M, Enomoto Y, Nishimatsu H, Kondo Y, Nagase Y, Hirano Y, Okaneya T, Tanaka Y, Miyazaki H, Fujimura T, Fukuhara H, Kume H, Igawa $\mathrm{Y}$ and Homma Y: Oncologic outcome of metastasectomy for urothelial carcinoma: who is the best candidate. Ann Surg Onco 24: 2794-2800, 2017. PMID: 28687875. DOI: 10.1245/s10434-017-5970-8 
22 Necchi A, Sonpavde G, Lo Vullo S, Giardiello D, Bamias A, Crabb SJ, Harshman LC, Bellmunt J, De Giorgi U, Sternberg CN, Cerbone L, Ladoire S, Wong YN, Yu EY, Chowdhury S, Niegisch G, Srinivas S, Vaishampayan UN, Pal SK, Agarwal N, Alva A, Baniel J, Golshayan AR, Morales-Barrera R, Bowles DW, Milowsky MI, Theodore C, Berthold DR, Daugaard G, Sridhar SS, Powles T, Rosenberg JE, Galsky MD, Mariani L and RISC Investigators: Nomogram-based prediction of overall survival in patients with metastatic urothelial carcinoma receiving first-line platinum-based chemotherapy: Retrospective international study of invasive/advanced cancer of the urothelium (RISC). Eur Urol 71: 281-289. 2017. PMID: 27726966. DOI: $10.1016 /$ j.eururo.2016.09.042

23 Ramos JD, Cheng HH and Yu EY: Long-term survival in bonepredominant metastatic urothelial carcinoma. Clin Genitourin Cancer 12: e241-244, 2014. PMID: 25160520. DOI: 10.1016/ j.clgc.2014.07.005

24 Pasquier J, Abu-Kaoud N, Al Thani H and Rafii A: Epithelial to mesenchymal transition in a clinical perspective. J Oncol 2015: 792182, 2015. PMID: 26425122. DOI: 10.1155/2015/792182

25 Bellomo C, Caja L and Moustakas A: Transforming growth factor $\beta$ as regulator of cancer stemness and metastasis. Br J Cancer 115: 761-769, 2016. PMID: 27537386. DOI: 10.1038/ bjc. 2016.255

26 Punyavoravut V and Nelson SD: Diffuse bony metastasis from transitional cell carcinoma of urinary bladder: a case report and review of literature. J Med Assoc Thai 82: 839-843, 1999. PMID: 10511795.

27 Rybinski B, Franco-Barraza J, Cukierman E: The wound healing, chronic fibrosis, and cancer progression triad. Physiol Genomics 46: 223-244, 2014. PMID: 24520152. DOI: 10.1152/ physiolgenomics.00158.2013

28 Alkasalias T, Moyano-Galceran L, Arsenian-Henriksson M and Lehti K: Fibroblasts in the tumor microenvironment: shield or spear? Int J Mol Sci 19 pii: E1532, 2018. PMID: 29883428. DOI: $10.3390 /$ ijms 19051532

29 Tao L, Huang G, Song H, Chen Y and Chen L: Cancer associated fibroblasts: An essential role in the tumor microenvironment. Oncol Lett 14: 2611-2620, 2017. PMID: 28927027. DOI: 10.3892/ol.2017.6497

30 LeBleu VS and Kalluri R: A peek into cancer-associated fibroblasts: origins, functions and translational impact. Dis Model Mech 11: dmm029447, 2018. PMID: 29686035. DOI: $10.1242 / \mathrm{dmm} .029447$

31. Candido J and Hagemann T: Cancer-related inflammation. J Clin Immunol 33(Suppl 1): S79-84, 2013. PMID: 23225204. DOI: $10.1007 / \mathrm{s} 10875-012-9847-0$

32. Fukuda K, Kobayashi A and Watabe K: The role of tumorassociated macrophage in tumor progression. Front Biosci (Schol Ed) 4: 787-798, 2012. PMID: 22202090.

33 Allavena P, Sica A, Solinas G, Porta C and Mantovani A: The inflammatory micro- environment in tumor progression: the role of tumor-associated macrophages. Crit Rev Oncol Hematol 66: 1-9, 2008. PMID: 17913510. DOI: 10.1016/j.critrevonc. 2007. 07.004
34 Diakos CI, Charles KA, McMillan DC and Clarke SJ: Cancerrelated inflammation and treatment effectiveness. Lancet Oncol 15: e493-503, 2014. PMID: 25281468. DOI: 10.1016/S14702045(14)70263-3

35 Ribatti D and Tamma R: A revisited concept. tumors: wounds that do not heal. Crit Rev Oncol Hematol 128: 65-69, 2018. PMID: 29958632. DOI: 10.1016/j.critrevonc.2018.05.016

36 Meng $\mathrm{X}$ and Riordan NH: Cancer is a functional repair tissue. Med Hypotheses 66: 486-490, 2006. PMID: 16290925. DOI: 10.1016/j.mehy.2005.09.041

37 Behrenbruch C, Shembrey C, Paquet-Fifield S, Mølck C, Cho HJ, Michael M, Thomson BNJ, Heriot AG and Hollande F: Surgical stress response and promotion of metastasis in colorectal cancer: a complex and heterogeneous process. Clin Exp Metastasis 35: 333-345, 2018. PMID: 2933581. DOI: 10.1007/s10585-018-9873-2

38 Zhang N, Wang L, Chai ZT, Zhu ZM, Zhu XD, Ma DN, Zhang QB, Zhao YM, Wang M, Ao JY, Ren ZG, Gao DM, Sun HC and Tang ZY: Incomplete radiofrequency ablation enhances invasiveness and metastasis of residual cancer of hepatocellular carcinoma cell HCCLM3 via activating $\beta$-catenin signaling. PLoS One 9: e115949, 2014. PMID: 25542041. DOI: 10.1371/ journal.pone.0115949

39 Alieva M, van Rheenen J and Broekman MLD: Potential impact of invasive surgical procedures on primary tumor growth and metastasis. Clin Exp Metastasis 35: 319-331, 2018. PMID: 28778871. DOI: 10.1101/cshperspect.a028662

40 Galdiero MR, Marone G and Mantovani A: Cancer inflammation and cytokines. Cold Spring Harb Perspect Biol 10 pii: a028662, 2018. PMID: 28778871. DOI: 10.1101/cshperspect.a028662

41 Landskron G, De la Fuente M, Thuwajit P, Thuwajit C and Hermoso MA: Chronic inflammation and cytokines in the tumor microenvironment. J Immunol Res 2014: 149185, 2014. PMID: 24901008. DOI: 10.1155/2014/149185

42 Morton LM, Dores GM, Tucker MA, Kim CJ, Onel K, Gilbert ES, Fraumeni JF Jr. and Curtis RE: Evolving risk of therapyrelated acute myeloid leukemia following cancer chemotherapy among adults in the United States, 1975-2008. Blood 121: 29963004, 2013. PMID: 23412096. DOI: 10.1182/blood-2012-08448068

43 Whatley WS, Thompson JW and Rao B: Salivary gland tumors in survivors of childhood cancer. Otolaryngol Head Neck Surg 134: 385-388, 2006. PMID: 16500432. DOI: 10.1016/j.otohns. 2005.10 .022 